\title{
The Effect of the Initial Rock Permeability on the Extent of Injectivity Reduction Due to Brine Injection through Fractured Formation
}

\author{
E.S. Al-Homadhi ${ }^{1}$ \\ 1 Petroleum Engineering Department, King Saud University, PO Box 800, Riyadh 11421 - Saudi Arabia \\ e-mail: ehomadhi@ksu.edu.sa
}

\begin{abstract}
Résumé - L'effet de la perméabilité initiale sur l'importance de la réduction d'injectivité due à l'injection d'eau salée au travers de formations fracturées - La réduction d'injectivité au cours du temps est un facteur important dans la définition des projets d'injection d'eau. Un des facteurs principaux affectant l'injectivité, en raison de l'invasion de particules, est la perméabilité initiale du milieu. Ce facteur a été étudié expérimentalement, et évalué par de nombreux chercheurs, mais tous ces travaux expérimentaux reposaient sur des essais d'écoulement linéaire.
\end{abstract}

Cependant, dans certains projets, la réduction d'injectivité au cours du temps est bien moindre que celle prévue par les modèles expérimentaux. Cette incompatibilité est liée à l'apparition de fractures causées par l'injection à une pression supérieure à la pression de fracturation de la formation.

Cette étude a été réalisée pour évaluer expérimentalement l'effet de la perméabilité initiale de la matrice sur la réduction de l'injectivité causée par l'injection d'eau salée dans une matrice rocheuse avec une seule fracture. L'eau salée injectée contient soit des particules solides jusqu'à $6 \mu \mathrm{m}$ de diamètre, soit des particules solides jusqu'à $20 \mu \mathrm{m}$, à une concentration de $9 \mathrm{mg} / \mathrm{l}$.

Les premiers résultats montrent expérimentalement une différence considérable de l'étendue de la réduction d'injectivité selon que les essais d'injection sont réalisés à fracture ouverte ou à fracture fermée.

Dans la suite, on présente l'indice d'injectivité à volume de pore injecté donné en fonction de la perméabilité initiale. La pente de cette courbe est appelée taux de réduction. Ce taux a été évalué pour différents cas. Dans le cas des suspensions salées contenant les petites particules, le taux de réduction de l'indice d'injectivité dû à la variation de la perméabilité initiale a été nettement moindre dans les essais à fracture ouverte que celui obtenu dans les essais à fracture fermée.

Dans le cas de l'injection de suspensions contenant les grosses particules, le taux de réduction a été trois fois plus grand que celui obtenu avec les suspensions de petites particules. Cela prouve que la taille de particule est un paramètre qui joue un rôle important dans la détermination de l'étendue de l'effet de la perméabilité initiale de la roche sur l'indice d'injectivité lors d'injection dans des fractures.

Mots-clés : injection d'eau, dommage de formation, perméabilité modifiée, fracture induite.

Abstract - The Effect of the Initial Rock Permeability on the Extent of Injectivity Reduction Due to Brine Injection through Fractured Formation - The injectivity reduction with time is an important aspect in designing water injection projects. One of the main factors that affect the injectivity, due to particle invasion, is the matrix initial permeability. This factor had been experimentally investigated and evaluated by many researchers, but all of their experimental works were based on linear core flow tests. 
However, in some field injection projects, there was a much less reduction in the injectivity with time than what was predicted by the experimental models. This incompatibility was related to the induction of fractures caused by injection at a pressure higher than the formation fracturing pressure.

This study was conducted to investigate experimentally the effect of the matrix initial permeability on the extent of injectivity reduction caused by brine injection through a rock matrix with a single fracture. The injected brine contained solid particles less than $6 \mu \mathrm{m}$ or less than $20 \mu \mathrm{m}$ in size at a concentration of $9 \mathrm{mg} /$.

The early results show experimentally the huge difference in the injectivity reduction extent between flow tests carried out with closed and open fracture injection.

Then, the results are presented as an injectivity index at a certain injected pore volume versus initial permeability. The slope of this relation is called reduction rate. This rate was evaluated for different cases. For the case of brine suspensions that contain small particles, the reduction rate of the injectivity index due to initial permeability variation in the case of open fracture tests was much less than that of the closed fracture tests.

For the case of fracture injection of the large particles suspensions, the reduction rate was threefold higher than that of the small particles suspension. This proves that the particle size factor has an important role in determining the extent of the initial rock permeability effect on the injectivity index in fracture injection.

Keywords: water injection, formation damage, permeability alteration, induced fractures.

\section{INTRODUCTION}

The rock matrix plugging extent, caused by brine flow through a porous medium, depends on:

- the size and concentration of solid particles contained in this brine;

- the pore-size distribution, porosity, and permeability of the porous medium;

- the nature of the interaction of the particles and the injected fluid with the rock materials and pore fluids;

- the flow velocity of the injected brine;

- the geometry of the injection system.

All of these factors have been studied by many researchers for many years and they came out with many conclusions. The effect of the initial matrix permeability is one of the main factors that were given big attention in literature.

Vetter et al. (1987) studied the damaging effect of brine flow through a porous medium having an initial permeability less than $200 \mathrm{mD}$. They concluded that if injected brine is filtered down to the lowest particle size, particles will still be deposited in the rock matrix if its permeability is in the order of 100 and even $200 \mathrm{mD}$.

Van Velzen and Leerlooijer (1992) carried out a set of linear and radial core flow experiments, using various particle sizes suspensions, to evaluate the effect of the particle/pore size ratio on permeability impairment (where pore size can be taken as a representative of initial rock permeability). For inflow velocity above $10 \mathrm{~cm} / \mathrm{min}$, they confirmed the generally accepted 1/3-1/7 rule giving the particle/pore size ratio for internal cake formation.

Todd et al. (1984) demonstrated local permeability impairment as a function of injected particles size and rock properties. They pointed out that initial permeability or pore size are not simple criteria for explaining the degree of damage to the cores. Shape and surface characteristics may also play an important role in trapping mechanisms.

Roque et al. (1995) experimentally demonstrated the different phases in the progressive plugging of a sandstone porous medium, of different permeabilities, subjected to the injection of particle suspensions. One of their conclusions is that for nearly the same pore throat size of $17 \mu \mathrm{m}$, increasing the injected particles size from 0.8 to $2.35 \mu \mathrm{m}$ causes an early and sharp loss in permeability.

Eleri and Ursin (1992) investigated the pressure profile for three different classes of porous medium having different initial permeabilities injected with $5 \mu \mathrm{m}$ particle suspensions. Pressure trends show an early and rapid pressure build-up in the case of medium-permeability core, near vertical for lowpermeability core, and a very low pressure increase for the case of high-permeability core.

Ershaghi et al. (1986) measured injectivity losses on a small scale duration, radial flow injection and demonstrated the dependency of the half life on both the rock initial permeability and particle-size distribution. By measuring particle counts in the effluents, they observed that for lowpermeability core most of the small pores were invaded and bridged by fine particles, whereas for high-permeability core, because of blockage of small pores, larger pores were still transmitting a considerable number of particles.

Pautz et al. (1989) classified the results of their core flood tests by the ratio of invading particle size to the square root of initial permeability. They indicated that using an average particle size to the square root of permeability ratio around or less than $1 / 5$ is appropriate for least damage. 
All the above studies were experimental core flow tests. In field tests, however, injectivity losses, when observed, occur over periods of weeks to months, and are inconsistent with the results of core flood studies. Simpson and Paige (1991) stated that no decline in well injectivity was observed throughout a six weeks' field injection trial. This was explained by fracturing and other forms of deformation caused by the injection process.

Fracturing increases injectivity, and creates a large sandface area, which takes a much longer time to plug. In some circumstances, fractures are capable of extension to create a new sand-face and maintain injectivity in response to any plugging which does occur.

Fracturing of water injection wells was studied and discussed by many authors, but a very few experimental works were carried out to simulate such a field case. One of the extended attempts to experimentally simulate such a case was carried out by Al-Homadhi (1998) at Heriot-Watt University. In his work an experimental fracture model was designed and constructed. The results were used to predict a simple mathematical model to simulate the damage extent for many injected brines having different compositions.

However, in this extensive study of fractured systems, the effect of matrix initial permeability variation on the injectivity decline extent due to brine injection, containing particles of constant size and concentration, was not studied, although he recommended studying such an important effect.

This paper contains an experimental work, which confirms the previous experimental work on the effect of the initial permeability on the damage extent in normal injection systems (closed fracture systems), and contains first-time experimental results of the effect of the initial permeability on the matrix damage extent in open fracture systems.

\section{OBJECTIVES OF THIS STUDY}

The general objective of this work is to quantify the effect of fracture initiation on the injectivity index versus initial permeability variation by:

- observing the injectivity reduction profile in the two systems (open and closed fracture injection systems) caused by pore plugging;

- measuring the reduction rate of the injectivity index due to initial rock permeability variation for the two systems;

- investigating the effect of the size of the particles contained in the injected brine, and of the number of injected pore volumes on the reduction rate value.

\section{EXPERIMENTAL DESIGN}

$20 \mathrm{~cm}$ long by $6 \mathrm{~cm}$ wide Berea sandstone slices with $1.5 \mathrm{~cm}$ thickness were cut, then these samples were fractured along their length into two halves. These samples have an average porosity of $16 \%$.

To simulate open fracture injection, these two halves were separated by a $0.5 \mathrm{~mm}$ spacer, the injection was directed along the fracture inlet and the production was collected along the sandstone sides and at the fracture end (Fig. 1). For the simulation of closed fracture injection, these two halves were pressed together to form one unit, the injection was directed through the $6 \times 1.5 \mathrm{~cm}$ face and the production through the opposite face while no flow was allowed through the sandstone sides. For both cases, no flow was allowed through the upper and lower sandstone faces.

The no-flow boundaries were glued into thick plastic sheets by araldite resin, and to prevent any fluid bypass a confining pressure was applied on these surfaces.

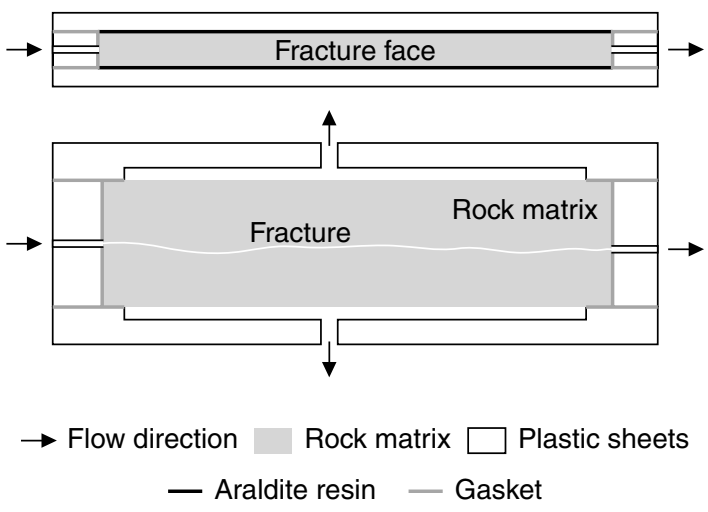

Figure 1

Top and side cross-sections through the open fracture system model.

\subsection{Flow Rate}

The injection rate was held constant, around $3.6 \mathrm{cc} / \mathrm{s}$, which is equivalent to a $2000 \mathrm{~m}^{3} / \mathrm{d}$ or $13000 \mathrm{bbl} / \mathrm{d}$ injection through a wellbore of $17.78 \mathrm{~cm}$ in diameter and $10.065 \mathrm{~m}$ formation thickness.

The flow velocity through the sandstone matrix for the case of closed fracture injection was $0.4 \mathrm{~cm} / \mathrm{s}$. For the case of open fracture injection, the flow velocity through the rock matrix was $0.024-0.058 \mathrm{~cm} / \mathrm{s}$.

\subsection{Injected Fluid}

The injected fluid was a $3.5 \% \mathrm{NaCl}$ brine solution containing alumina particles with a concentration of $9 \mathrm{mg} / \mathrm{l}$.

Two groups of alumina particles were used:

- the small particles group had a distribution of 0.1-6 $\mu \mathrm{m}$ with $80 \%$ of the particles being less than $3 \mu \mathrm{m}$ in size;

- the large particles group had a distribution of $0.1-20 \mu \mathrm{m}$ with $80 \%$ of the particles being less than $8 \mu \mathrm{m}$ in size. 
These two particles groups and their concentration were chosen to be compatible with the reported downhole injected water quality in actual injectors. Hsi and Strassner (1990) reported particle-size analysis of Prudhoe injected seawater. Nearly $84 \%$ of the particles were less than $3 \mu \mathrm{m}$ in size with a wide particle-size distribution from 0 to $10 \mu \mathrm{m}$. Clifford $e t$ al. (1991) reported particle-size analysis of Prudhoe and Ula on a downhole injected with produced water. Nearly $98 \%$ of the particles were less than $8 \mu \mathrm{m}$ in size with a wide particlesize distribution from 0 to $16 \mu \mathrm{m}$. Also, they reported a downhole particle concentration of less than $8 \mathrm{ppm}$.

To enhance particle dispersion, the particle suspension was placed in an ultrasonic path before it was added to the bulk brine solution.

A circulation pump was connected to the main injection tank to keep the particles suspended and well dispersed within the aqueous phase (Fig. 2).

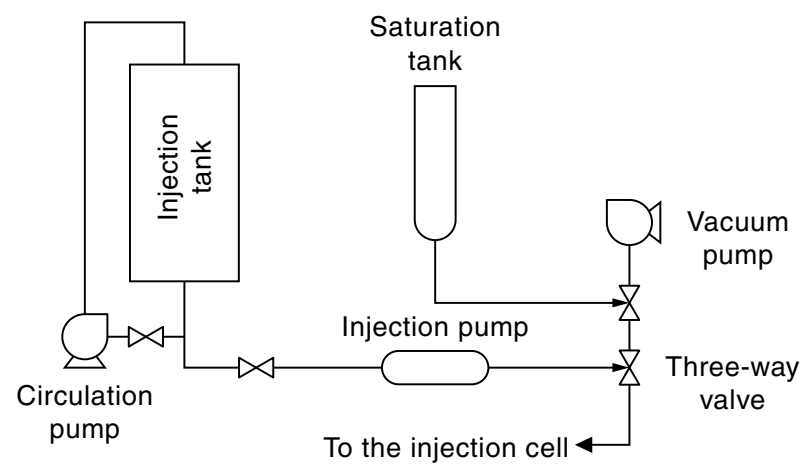

Figure 2

Schematic diagram of the injection rig.

\subsection{Injectivity Test}

At the start of each test, the sandstone sample was vacuumed and saturated with filtered salt water. Then, the initial permeability was measured by flowing filtered salt water through the sample and applying Darcy's law.

When brine injection was initiated, pressure drop started to increase due to particle invasion.

\subsubsection{For the Case of Open Fracture Injection}

Pressure drop equals the average gauge pressure at the fracture face, as sandstone sides were kept at atmospheric pressure. The average pressure was measured by knowing the pressure drop along the fracture. The flow rate equals the total collected flow rates through the sandstone two lateral side outlets.

This system simulates the actual fractured near-wellbore condition (the first $20 \mathrm{~cm}$ adjacent to the well wall), and not the flow conditions at the fracture tip.

\subsubsection{For the Case of Closed Fracture Injection}

As most of matrix damage occurs within the first few centimeters near the injection face, and as the flow path in the open fracture was only $3 \mathrm{~cm}$ (which equals half width of the fractured rock), only pressure drop across the first $3 \mathrm{~cm}$ was taken into account and used to calculate injectivity.

\section{RESULTS AND DISCUSSION}

\subsection{Particle-Size Analysis}

The studied case in this paper represents an injection of low particle concentration suspensions. No external cake was observed on the open fracture face or on the closed fracture flow surface.

Table 1 shows particle analysis of the fracture outlet flow. For the first size distribution, the mean size $\left(D_{E}\right)$ increased from $1.7 \mu \mathrm{m}$ to reach the same size as the injected particles at the end of tests. The $C_{E} / C_{0}$ (fracture outlet flow particle concentration/injected particles initial concentration) was between 0.8 and 1 . For the second size distribution, the mean size increased from $3 \mu \mathrm{m}$ to reach $4.5 \mu \mathrm{m}$ at the end of tests. And the $C_{E} / C_{0}$ was between 0.6 and 0.93 .

TABLE 1

Particles mean size and concentration analysis of the fracture outlet flow

\begin{tabular}{c|c|c}
\hline $\begin{array}{c}\text { Mean size } \\
\text { of the injected } \\
\text { particles }(\mu \mathrm{m})\end{array}$ & $\begin{array}{c}\text { Particles mean } \\
\text { size of the open } \\
\text { fracture outlet } \\
\text { flow, } D_{E}(\mu \mathrm{m})\end{array}$ & $\begin{array}{c}\text { Particle concentration } \\
\text { of the open fracture } \\
\text { outlet flow/initial } \\
\text { concentration, } C_{E} / C_{0}\end{array}$ \\
\hline 2.3 & $1.7-2.3$ & $1-0.8$ \\
5 & $3-4.5$ & $0.93-0.6$ \\
\hline
\end{tabular}

The particles at the fracture outlet had lower mean size and concentration than the injected particles. This indicates that particles (sometimes with larger mean size) in a concentration higher than the injected flow are directed toward the fracture face and into the rock matrix. This makes the open fracture system have the same chance (probability) of undergoing the same matrix damage mechanisms as the closed fracture system. Furthermore, and due to the very low flow velocity through the rock matrix, the matrix in the open fracture system may receive a higher damage than the matrix in the closed fracture system. This can be clearly seen from the results of the outlet flow size analysis in the two systems. In the open fracture, the $C_{S} / C_{0}$ (lateral flow particle concentration/injected particles initial concentration) was less than 0.35 , while in the closed fracture the $C / C_{0}$ (closed fracture effluent particle concentration/injected particles initial concentration) was between 0.6 and 0.75 (Table 2). 
TABLE 2

Particles mean size and concentration analysis of the outlet flow through the rock matrix

\begin{tabular}{c|c|c|c|c}
\hline $\begin{array}{c}\text { Mean size } \\
\text { of the injected } \\
\text { particles }(\mu \mathrm{m})\end{array}$ & $\begin{array}{c}\text { Particles mean size } \\
\text { of the side outlet flow } \\
\text { in open fracture, } D_{S}(\mu \mathrm{m})\end{array}$ & $\begin{array}{c}\text { Particle concentration } \\
\text { of the side outlet flow } \\
\text { in open fracture/initial } \\
\text { concentration, } C_{S} / C_{0}\end{array}$ & $\begin{array}{c}\text { Particles mean size } \\
\text { of the outlet flow } \\
\text { in closed fracture, } \\
D(\mu \mathrm{m})\end{array}$ & $\begin{array}{c}\text { Particle concentration } \\
\text { of the outlet flow in } \\
\text { closed fracture/initial } \\
\text { concentration, } C / C_{0}\end{array}$ \\
\hline 2.3 & $0.4-2.3$ & $0-0.25$ & $1.74-1.9$ & $0.6-0.66$ \\
5 & $3-4.5$ & $0-0.35$ & $4-4.6$ & $0.64-0.75$ \\
\hline
\end{tabular}

In the open fracture system, the $C_{S} / C_{0}$ of the lateral outlet flow for the case of small particles (first distribution) injection was nearly similar to that of the large particles (second distribution). This may lead us to believe that (for the two distributions) the matrix has already passed through the same damage mechanisms (Table 2). The low flow velocity through the rock matrix in the open fracture system gives a weak driving force, which can drive and distribute small particles (first distribution) deep into the rock. But this weak driving force cannot effectively drive and distribute the large particles (second distribution). This leads in the case of the second distribution to large particles accumulation within the rock matrix close to the fracture face and consequently to a higher flow resistance.

\subsection{Injectivity Loss in the Two Systems}

Figures 3 and 4 show plots of injectivity loss versus pore volume injected for the two systems. The number of pore volume injected in the case of open fracture was calculated using the total outlet flow rate on the lateral sides only. The value of this flow rate depends on the initial permeability of the sandstone.
At the start of injection, the injectivity of the open fracture system was nearly six times higher than that of the closed fracture system. After injecting 2000 pore volumes, this difference increased to nearly nine times (Figs. 3 and 4). This occurred though the flow area of the open fracture injection (fracture face) was only 6.7 times larger than that of the closed fracture injection system, and though the flow velocity (through the rock matrix) in the closed fracture injection was ten times higher than that of the open fracture injection.

The difference in the injectivity between the two systems is due to the difference in the flow area and flow velocity. In the open fracture injection, the large flow area enhanced the injectivity, whereas the very low flow velocity may cause some reduction in the injectivity (as the driving force, which drives the solid particles deep into the rock matrix, is very small).

In open fracture systems, the large particles could cause much more pore bridging than small particles. The very low flow velocity resulted in a very weak driving force, which could only drive small particles deep into the matrix and spread the damage loss on the whole matrix pore volume. This reduced its effect on the injectivity. This driving force may not efficiently drive the large particles deep enough into

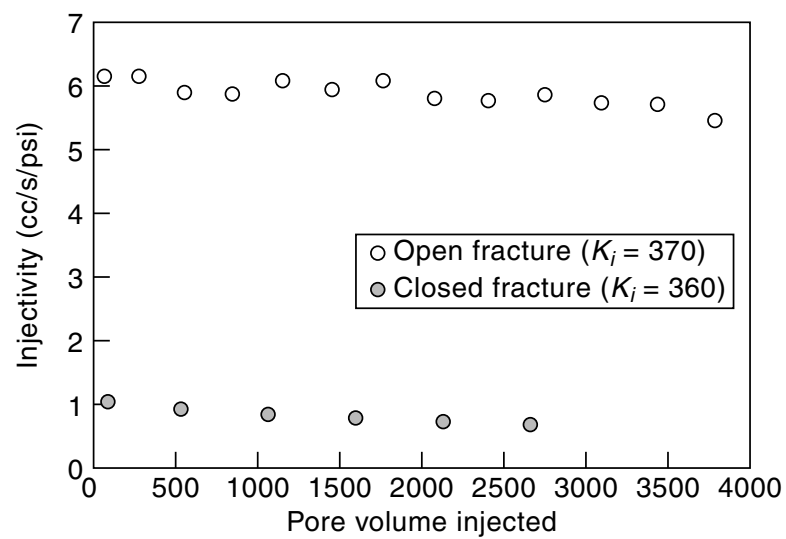

Figure 3

Injectivity reduction due to small particles injection.

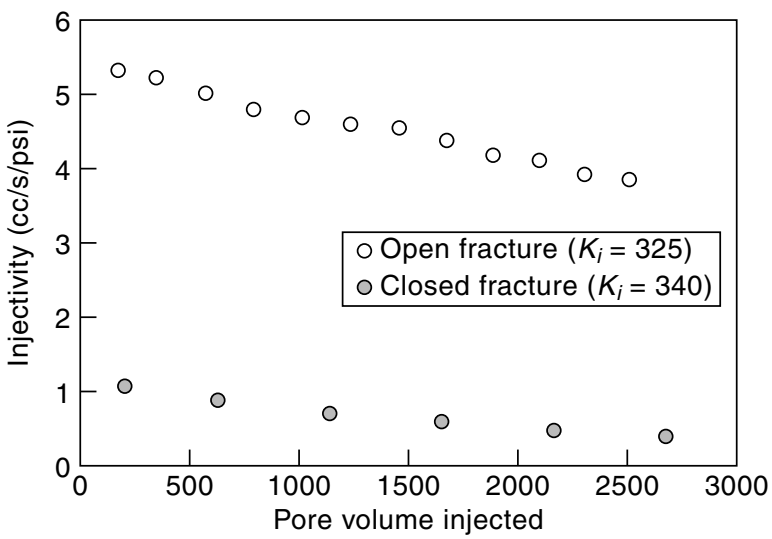

Figure 4

Injectivity reduction due to large particles injection. 
the rock matrix, which resulted in their accumulation within the matrix close to the fracture face and accordingly caused a higher loss in the injectivity.

\subsection{Effect of Initial Permeability on Injectivity Trend}

Figure 5 represents the injectivity loss in the open fracture system, caused by the small particles brine suspension, for two rock samples having different initial permeabilities. Permeability variation has a minimal effect on injectivity trend through the first 500 injected pore volumes. Increasing the injected pore volumes results in a small difference in the injectivity index between the two rock samples.

Figure 6 represents the injectivity loss in the open fracture system, caused by the large particles suspension, for two rock samples having different initial permeabilities. The difference in the injectivity loss between the two rock samples was obvious at the start of injection, and throughout the whole test duration.

Figures 7 and 8 show the injectivity loss for the closed fracture system. Reducing initial permeability in the closed fracture system causes higher reduction in the injectivity index than it does in the open fracture injection system.

These results indicate that for open fracture injection systems, reducing the size of the particles contained in the injected brine to less than $3 \mu \mathrm{m}$ (at a concentration of $9 \mathrm{mg} / \mathrm{l}$ ) insures a good injectivity for a long time and increases the lifetime of the injector.

\subsection{Injectivity Index versus Initial Permeability}

The loss in the injectivity due to initial rock permeability variation, at a constant brine particle content and at a certain number of injected pore volumes, will be called reduction

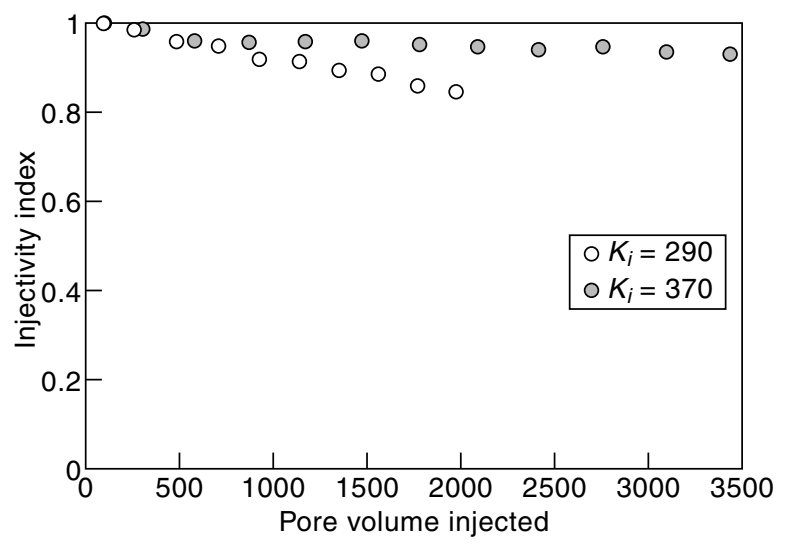

Figure 5

Injectivity index versus injected pore volumes for the small particles injection through open fracture systems.

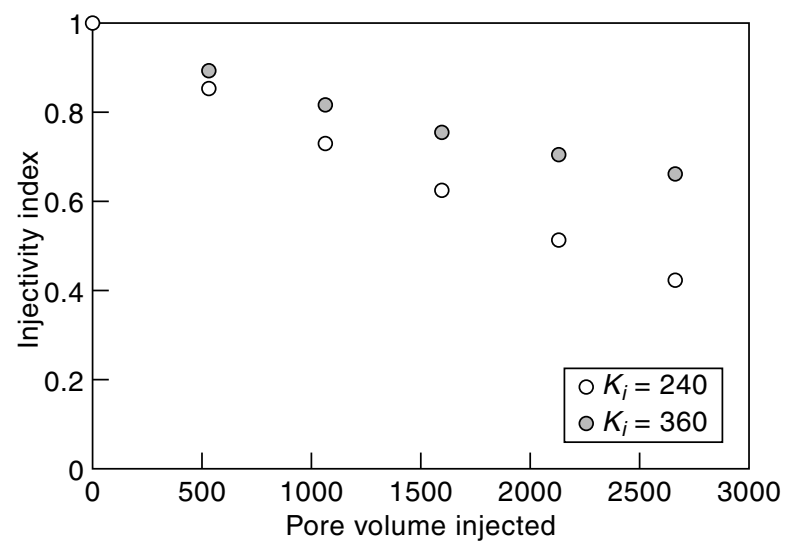

Figure 7

Injectivity index versus injected pore volumes for the small particles injection through closed fracture systems.

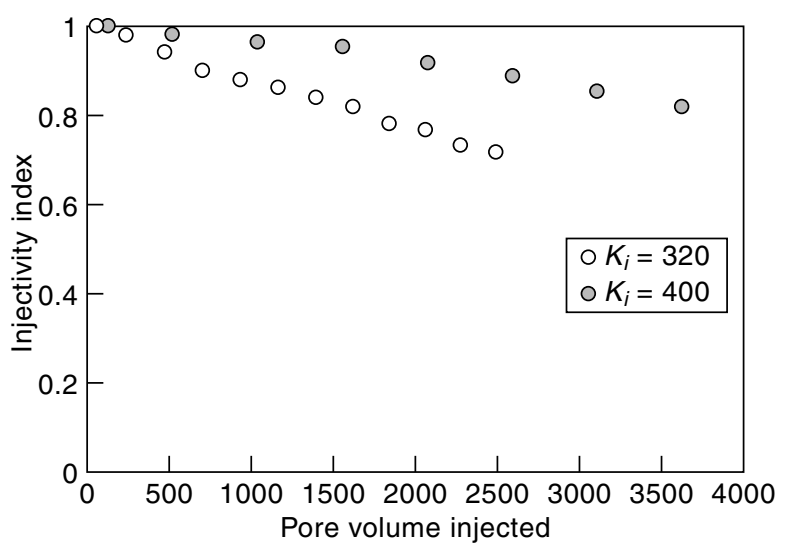

Figure 6

Injectivity index versus injected pore volumes for the large particles injection through open fracture systems.

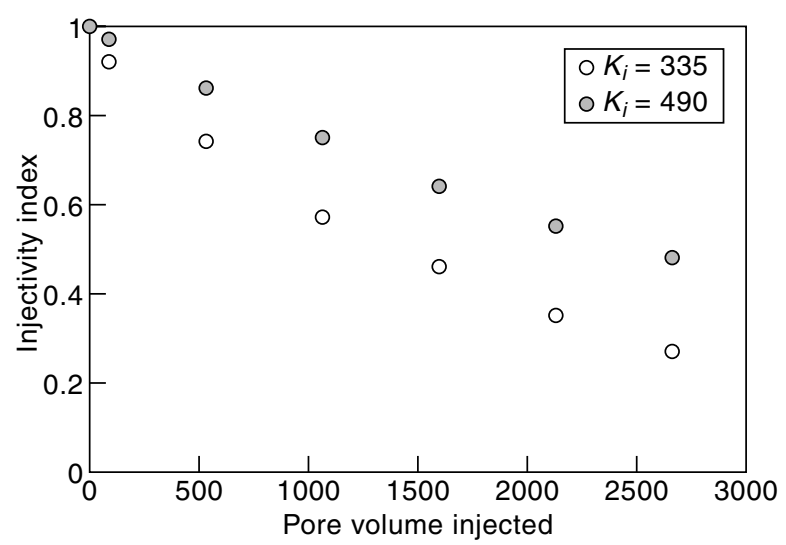

Figure 8

Injectivity index versus injected pore volumes for the large particles injection through closed fracture systems. 
rate. This rate equals the slope of the injectivity index versus initial permeability variation relationship.

The experimental results were presented as an injectivity index at 2000 injected pore volumes versus initial permeability (Figs. 9 and 10). Sandstones with initial permeabilities of 200 to $550 \mathrm{mD}$ were used to investigate the reduction rate in the two injection systems.

In general, the reduction rate was higher in the case of large particles injection than it was for the small particles, as can be seen from the slopes in Figures 9 and 10.

Concerning the injection tests of small particles suspensions:

- in open fracture injection systems, initial rock permeability variation had a minor effect on the injectivity index. On the other hand it had a higher effect on the injectivity index in the closed fracture injection system (Fig. 9);

- in the closed fracture injection, the reduction rate was more than three times higher than that of the open fracture injection tests.

Concerning the injection tests of large particles suspensions:

- for the case of open fracture injection, the reduction rate was nearly three times the reduction rate of small particles injection tests;

- for the case of closed fracture injection, the reduction rate was only $20 \%$ higher than that of small particles injection tests;

- the reduction rate in the case of open fracture becomes closer to the reduction rate of the closed fracture injection tests.

These results show that the reduction rate in the open fracture injection system is very sensitive to the variation of the injected particles size, whereas the closed fracture injection was less sensitive. For open fracture injection, increasing the injected particles size to the double trebles the effect of initial permeability on the injectivity index (or trebles the reduction rate).

In other words, for the open fracture injection, as the injected particles size increases, the initial permeability effectiveness in reducing the injectivity index increases sharply.

\subsection{The Variation of Reduction Rate with Pore Volume Injected}

Figures 11 to 14 show the injectivity index versus initial permeability variation (reduction rate) after injecting 500 and 2000 pore volumes.

\subsubsection{Open Fracture Injection Tests}

For the small particles injection, increasing the injected pore volume from 500 to 2000 gave a slight increase in the reduction rate (Fig. 11), whereas for the case of large particles injection, the reduction rate was doubled (Fig. 12).

\subsubsection{Closed Fracture Injection Tests}

At 500 pore volumes, the reduction rate due to small particles injection was equal to the reduction rate of the open fracture injection at 2000 pore volumes (Fig. 13). The same result was repeated for the large particles injection (Fig. 14).

This proves again the huge difference in the injectivity reduction extent, due to initial permeability variation, between closed and open fracture injection systems as an open fracture system can accommodate larger injected pore volumes without causing a serious injectivity reduction.

The reduction rate is function of injected pore volumes. It increases as injected pore volumes increase. As the injected pore volumes increase, the low initial permeability matrix suffers, due to its smaller pore throat, a higher pore bridging than the high initial permeability matrix.

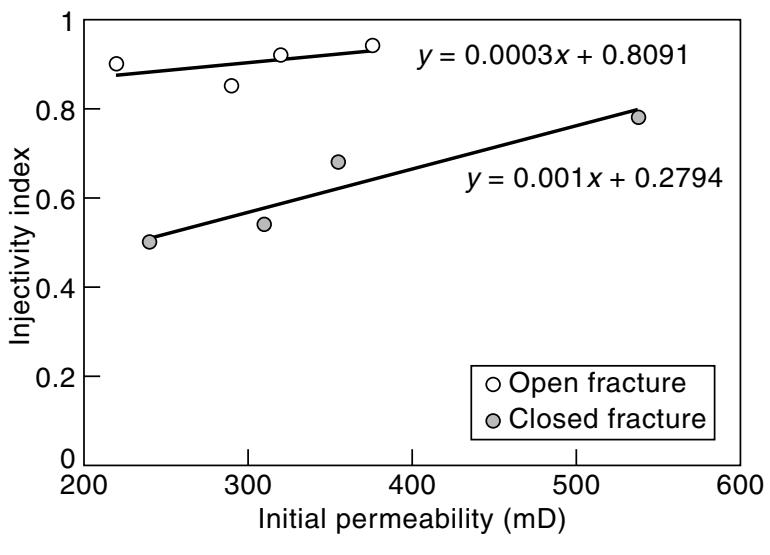

Figure 9

Injectivity index versus initial permeability for the small particles injection tests at 2000 injected pore volumes.

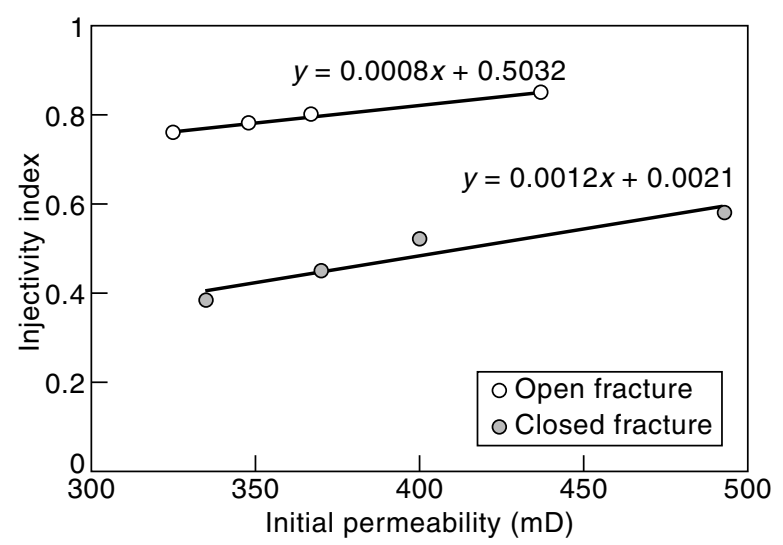

Figure 10

Injectivity index versus initial permeability for the large particles injection tests at 2000 injected pore volumes. 


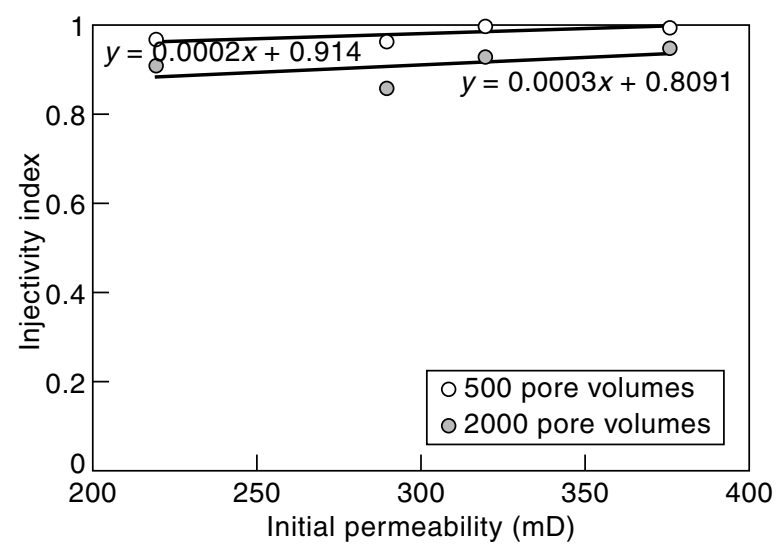

Figure 11

Reduction rate of small particles injection through open fracture systems at two injected pore volumes.

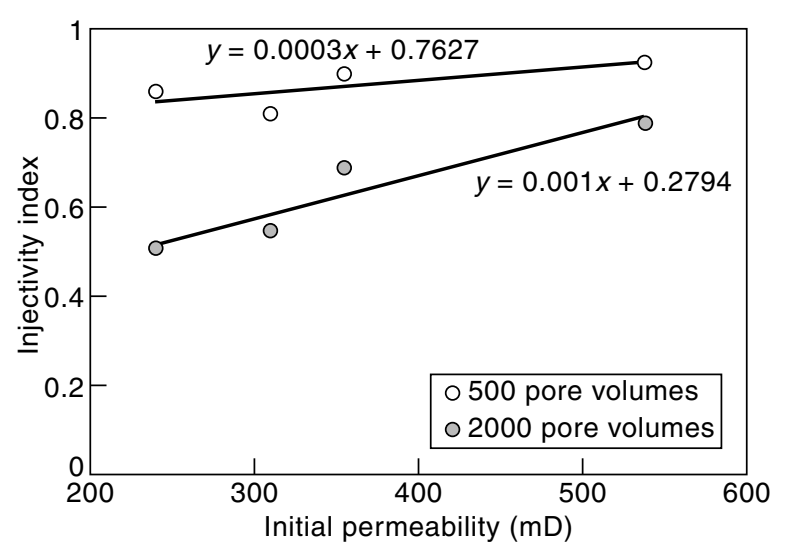

Figure 13

Reduction rate of small particles injection through closed fracture systems at two injected pore volumes.

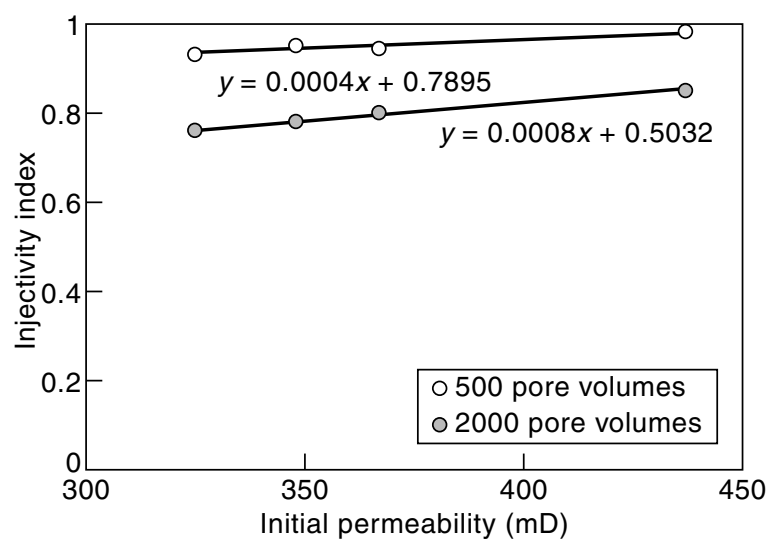

Figure 12

Reduction rate of large particles injection through open fracture systems at two injected pore volumes.

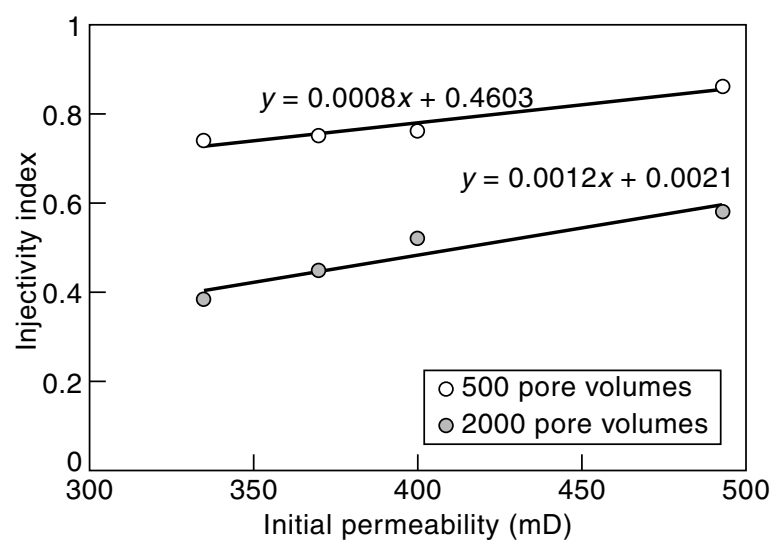

Figure 14

Reduction rate of large particles injection through closed fracture systems at two injected pore volumes.
For the case of small particles injection, this increase was much higher in the closed fracture injection than it was in open fracture injection tests. For the case of large particles injection, the opposite trend was observed. These results indicate again the sensitivity of the open fracture injection to the injected particles size variation.

\section{CONCLUSIONS}

In the open fracture injection, the large flow area enhances the injectivity, whereas the very low flow velocity through the rock matrix causes some reduction in the injectivity.

At the start of injection, the injectivity for the fracture system was nearly six times higher than that of the closed fracture system. After injecting 2000 pore volumes, this difference increases to nine times.
In fracture injection systems, reducing the size of the particles contained in the injected brine to less than $3 \mu \mathrm{m}$ insures a good injectivity for a long time.

For the tested injected particle-size distribution, a lower initial rock permeability can cause a higher injectivity loss in the closed fracture system than it does in the open fracture system.

For the tested injected particle-size distributions, the reduction rate in the open fracture injection system is very sensitive to the variation in the size of the injected particles, whereas the closed fracture system is less sensitive. The driving force of the flow in the case of closed fracture can flush particles, having a mean size of and less than $5 \mu \mathrm{m}$, deep into the formation. But in the open fracture case, the low flow velocity produces a weak driving force with less ability to drive these particles deep into the formation, 
resulting in their accumulation within the rock matrix near to the fracture face and causing higher flow resistance.

At 500 injected pore volumes, the reduction rate of closed fracture injection, due to initial permeability variation, was equal to the reduction rate of the open fracture injection at 2000 pore volumes.

The reduction rate is function of injected pore volumes.

\section{REFERENCES}

Al-Homadhi, E.S. (1998) A Study of the Formation Damage Associated with the Injection of Oil and Solid Suspensions into Fractured and Non-Fractured Rock. PhD Thesis, Department of Petroleum Engineering, Heriot-Watt University, Edinburgh, Scotland.

Clifford, P.J., Mellor, D.W. and Jones, T.J. (1991) Water Quality Requirement for Fractured Injection Wells. SPE Middle East Oil Show, Bahrain, November, SPE 21439.

Eleri, O.O. and Ursin, J.R. (1992) Physical Aspects of Formation Damage in Linear Flooding Experiments. SPE International Symposium on Formation Damage Control, Lafayette, Louisiana, February, SPE 23784.

Ershaghi, I., Hashemi, R., Caothien, S.C. and Abdassah, D. (1986) Injectivity Loss under Particle Cake Buildup and Particle Invasion. The SPE 56th California Regional Meeting, Oakland, California, April, SPE 15073.
Hsi, C.D. and Strassner, J.E. (1990) Prudhoe Bay Field, Alaska, Waterflood Injection Water Quality and Remedial Treatment Study. The SPE 65th Annual Technical Conference, New Orleans, Louisiana, September, SPE 20689.

Pautz, J.F., Crocker, M.E. and Walton, C.G. (1989) Relating Water Quality and Formation Permeability to Loss of Injectivity. The SPE Production Operations Symposium, Oklahoma, March, SPE 18888.

Roque, C., Chauveteau, G., Renard, M., Thibault, G., Bouteca, M. and Rochon, J. (1995) Mechanisms of Formation Damage by Retention of Particles Suspended in Injection Water. The European Formation Damage Conference, Netherlands, May, SPE 30110.

Simpson, A.J. and Paige, R.W. (1991) Advances in Forties Field Water Injection. The Offshore Europe Conference, Aberdeen, Scotland, September, SPE 23140.

Todd, A.C., Somerville, J.E. and Graham, S. (1984) The Application of Depth of Formation Damage Measurement in Predicting Water Injectivity Decline. Formation Damage Control Symposium, Bakersfield, California, February, SPE 12498.

Van Velzen, J.F. and Klaas, L. (1992) Impairment of a Water Injection Well by Suspended Solids: Testing and Prediction. The SPE International Symposium on Formation Damage Control, Lafayette, Louisiana, February, SPE 23822.

Vetter, O.J., Kandarpa, V., Stratton, M. and Veith, E. (1987) Particle Invasion into Porous Medium and Related Injectivity Problems. The SPE International Symposium on Oilfield Chemistry, San Antonio, Texas, February, SPE 16255.

Final manuscript received in January 2001 